\title{
Evaluación de factores cognitivos positivos y negativos relacionadas con el trastorno de pánico: Validación del CATP
}

\author{
Carmen Sánchez-Arribas ${ }^{1}$, Paloma Chorot ${ }^{2}$, Rosa M. Valiente ${ }^{2}$ y Bonifacio Sandín ${ }^{2}$ \\ ${ }^{1}$ Hospital General de Úbeda, Jaén, España \\ ${ }^{2}$ Universidad Nacional de Educación a Distancia (UNED), Madrid, España
}

\begin{abstract}
Resumen: El objetivo del estudio fue investigar la estructura factorial y las propiedades psicométricas del Cuestionario Abreviado del Trastorno de Pánico (CATP) y, adicionalmente, de la Panic Disorder Severity Scale-Self Report (PDSS-SR), en una muestra de pacientes con diagnóstico principal de trastorno de pánico (TP). El CATP incluye tres escalas breves diseñadas para evaluar constructos cognitivos relacionados con el TP, i.e., Escala de Interpretaciones Catastrofistas al Pánico (EICP), Escala de Autoeficacia ante el Pánico (EAP) y Escala de Gravedad del Pánico (EGP). Los análisis factoriales exploratorios indicaron una estructura trifactorial de la escala EICP consistente con las tres dimensiones de interpretaciones catastrofistas (física, mental y social). Las restantes escalas (EAP, EGP y PDSS-SR) exhibieron una robusta estructura unidimensional. Los datos sobre fiabilidad (estructura interna) y validez (convergente y discriminante) muestran que las escalas poseen excelentes propiedades psicométricas y pueden resultar de utilidad para la evaluación de constructos positivos y negativos del TP.
\end{abstract}

Palabras clave: Trastorno de pánico; interpretaciones catastrofistas; autoeficacia; gravedad del pánico; evaluación; CATP; EICP; EAP; EGP; PDSS-SR.

\section{Assessment of positive and negative cognitive factors related to panic disorder: Validation of the CATP}

\begin{abstract}
The aim of the study was to examine the factor structure and psychometric properties of the Abbreviated Panic Disorder Questionnaire (CATP) and, in addition, the Panic Disorder Severity Scale-Self Report (PDSS-SR), in a sample of patients with a principal diagnosis of panic disorder (PD). The CATP comprises three separate short scales designed to assess cognitive constructs related to PD, being the Panic Catastrophic Misinterpretations Scale (PCMS), the Panic Self-efficacy Scale (PSES) and the Panic Severity Scale (PSS). Exploratory factor analyses showed a three-factor structure of the PCMS consistent with the three hypothesized dimensions of catastrophic misinterpretations, i.e., physical, mental and social. We also found a strong unidimensional structure for the remaining scales (PSES, PSS and PDSS). Data on reliability (internal consistency) and validity (convergent and discriminant) show that the scales have sound psychometric properties and could be useful instruments to assess positive and negative constructs related to PD.
\end{abstract}

Keywords: Panic disorder; catastrophic misinterpretations; self-efficacy; panic severity; assessment; CATP; PCMS; PSES; PSS; PDSS-SR.

\section{Introduction}

A lo largo de los últimos años se han venido produciendo diferentes enfoques teóricos sobre la etiopatogenia del trastorno de pánico y la ansiedad, enfatizándose

Recibido: 31 octubre 2014; aceptado: 30 enero 2015.

Correspondencia: Bonifacio Sandín, Facultad de Psicología, Universidad Nacional de Educación a Distancia, Juan del Rosal 10, 28040

Madrid, España. Correo-e: bsandin@psi.uned.es en cada uno de ellos algún concepto relevante, incluyendo las leyes del condicionamiento clásico (Wolpe y Rowan, 1988), la ley de incubación (Eysenck, 1985; Chorot, 1989, 1991; Chorot y Sandín, 1993; Sandín y Chorot, 1989), la hiperventilación (Klein, 1993), la autofocalización corporal (Bernstein et al., 2008), los factores neuroanatómicos (Gorman, Kent, Sullivan y Coplan, 2000), el aprendizaje inhibitorio (Torrents-Rodas et al., 2015), y los factores cognitivos (Clark, 1986; McNally, 1994). Para revisiones sobre el tema, véase San- 
dín y Chorot (1991), Bouton, Mineka y Barlow (2001), Fava y Morton (2009), y Pilecki, Arentoft y McKay (2011).

La conceptualización cognitiva del trastorno de pánico (TP) se inició a partir del influyente modelo descrito originalmente por Clark (1986), el cual se basa en las interpretaciones catastrofistas de las sensaciones corporales como elemento central determinante de la etiología y el mantenimiento de los ataques de pánico y del TP. La principal característica del modelo de Clark consiste en que el pánico se origina debido a la existencia de interpretaciones catastrofistas que el individuo hace ante la ocurrencia de sensaciones corporales benignas (p.ej., la ocurrencia de una aceleración en la frecuencia cardíaca puede ser interpretada como un inminente ataque cardíaco). Clark interpreta el concepto de "catástrofe" como un fenómeno inevitable e inmediato. Por tanto, la clave del modelo está en que ciertas personas llevan a cabo interpretaciones cognitivas erróneas y desadaptativas de las sensaciones de activación fisiológica (actividad cardíaca, respiración, etc.), dando lugar a una espiral que lleva a la ocurrencia y mantenimiento del ataque de pánico. Esta teoría ha sido extensamente aplicada en el ámbito clínico, y ha demostrado ser de utilidad para el tratamiento del TP (Craske y Barlow, 2008). Aunque algunos estudios no han proporcionado un claro apoyo a la asociación entre las interpretaciones catastrofistas de las sensaciones corporales y el TP (p.ej., Fenz et al., 2013), la evidencia clínica tiende a apoyar un papel predominante de dichas interpretaciones en la predicción de la gravedad del TP y en los mecanismos de cambio asociados a la terapia cognitivo-conductual aplicada al TP (Casey, Oei, Newcombe, y Kenardy, 2004b; Clark, 1999; Khawaja y Oei, 1998; Sandín, Sánchez-Arribas, Chorot, y Valiente, 2015). En una línea similar, se ha demostrado que los pacientes con TP son más propensos que los pacientes con otros trastornos mentales (incluidos los trastornos de ansiedad) a interpretar las sensaciones corporales como indicadores de catástrofes de tipo físico, mental o social (Austin y Richards, 2006; Clark et al., 1997; Khawaja y Oei, 1998).

Aunque el modelo cognitivo del Clark ha sido el modelo dominante sobre el pánico en la psicología clínica, algunos autores han enfatizado la necesidad de incorporar al modelo componentes cognitivos de tipo positivo. Específicamente, Casey, Oei, and Newcombe (2004a) incorporaron el concepto de auto-eficacia ante el pánico (un factor cognitivo de naturaleza positiva) como elemento fundamental en la psicopatología del TP. La "auto-eficacia ante el pánico" se refiere a la percepción que tiene el individuo sobre su capacidad para controlar un ataque de pánico (bien sea antes de su ocurrencia, o bien una vez que éste se ha iniciado). Basándose en dicho concepto, estos autores propusieron un nuevo modelo cognitivo que denominaron "modelo cognitivo integrativo del trastorno de pánico" (Casey et al., 2004a). De acuerdo con estos autores, tanto la presencia de interpretaciones catastrofistas de las sensaciones corporales como la ausencia de auto-eficacia ante el pánico contribuyen a la gravedad del TP, y ambas desempeñan un papel fundamental en la psicopatología del trastorno pánico. Algunas investigaciones recientes han proporcionado evidencia empírica en favor de esta hipótesis que integra en la etiopatogenia del pánico los factores negativos (i.e., interpretaciones catastrofistas) y los factores positivos (i.e., autoeficacia ante el pánico) (Casey et al., 2004b; Fenz et al., 2013; Gallagher et al., 2013; Richards, Richardson y Pier, 2002; Sandín et al., 2015).

El modelo cognitivo de Casey et al. (2004a) constituye un enfoque del pánico más integrativo que el establecido por Clark (1986), pero no integra el concepto de sensibilidad a la ansiedad, uno de los constructos cognitivos más relevantes relacionados con la psicopatología del pánico y del TP. La sensibilidad a la ansiedad es un concepto muy cercano al de interpretaciones catastrofistas. Fue descrito originalmente por Reiss y McNally (1985) como un constructo disposicional (variable de diferencias individuales) relacionado con la tendencia a experimentar miedo ante los síntomas de ansiedad (McNally, 1990, 1994; Sandín, Chorot, Valiente, Santed, y Lostao, 2004b; Taylor, 1995; 1999). Basándonos en estudios transversales y longitudinales, existe suficiente evidencia para indicar que la sensibilidad a la ansiedad se relaciona de forma positiva con: (a) el diagnóstico de TP, (b) indicadores de síntomas del TP, (c) desarrollo de nuevos ataques de pánico, (d) evolución de la gravedad del TP, y (e) cambios en la gravedad del TP durante el tratamiento basado en terapia cognitivo-conductual (Sandín et al., 2015).

Recientemente, Sandín et al. (2015), partiendo del modelo integrativo de Casey et al., 2004a), propusieron un nuevo modelo cognitivo del TP. Este modelo, denominado "modelo tripartito del trastorno de pánico", integra los tres constructos cognitivos fundamentales asociados a la etiopatogenia del pánico y del TP, esto es, la sensibilidad a la ansiedad, las interpretaciones catastrofistas, y la autoeficacia ante el pánico. Los autores proporcionaron evidencia empírica sobre la contribución diferencial de las tres variables en la predicción de la gravedad del TP (Sandín et al., 2015). El modelo se basa, así mismo, en la separación conceptual entre la sensibilidad a la ansiedad y las interpretaciones catastrofistas. Aunque ambos conceptos (sensibilidad a la ansiedad e interpretaciones catastrofistas) representan cogniciones 
negativas relacionadas con el miedo a los síntomas interoceptivos, algunos autores los han diferenciado sobre la base de distintos fenómenos, tales como la distinción entre rasgo (i.e., sensibilidad a la ansiedad) y estado (i.e., interpretaciones catastrofistas), y la no necesaria implicación de interpretaciones catastrofistas asociadas a la sensibilidad a la ansiedad (McNally, 1990, 1994, 2002; Taylor, 1995, 2000). Por ejemplo, la hipótesis de sensibilidad a la ansiedad asume que las personas con alta sensibilidad a la ansiedad pueden experimentar temor a las sensaciones corporales únicamente porque señalan la ocurrencia de pánico o miedo al pánico, pero no se asocian necesariamente a la ocurrencia de interpretaciones catastrofistas en el sentido descrito por Clark (McNally, 2002). Por tanto, una diferencia fundamental entre ambos constructos consiste en que la sensibilidad a la ansiedad puede actuar sobre los procesos del pánico, incluida la gravedad, sin la intervención de las atribuciones catastrofistas (Sandín et al., 2015). En esta investigación encontramos que la sensibilidad a la ansiedad, las interpretaciones catastrofistas y la autoeficacia ante el pánico predecían de forma independiente la gravedad del TP. Más aun, observamos que la sensibilidad a la ansiedad se relacionaba de forma directa e indirecta (mediado por las interpretaciones catastrofistas) con la gravedad del TP, proporcionando evidencia sobre la separación entre ambos constructos (i.e., sensibilidad a la ansiedad vs. interpretaciones catastrofistas) y apoyando empíricamente el modelo cognitivo tripartito del TP.

Partiendo del modelo tripartito del TP, debemos asumir el papel central de los tres componentes cognitivos nucleares del mismo, i.e., los conceptos de sensibilidad a la ansiedad, interpretaciones catastrofistas de los síntomas del ataque de pánico (o síntomas similares a los del ataque de pánico), y la autoeficacia ante el ataque de pánico. Sería importante, por tanto, disponer de instrumentos validados que posibiliten la evaluación de estos tres constructos. Esto resulta de particular interés, tanto para fines de investigación como para la práctica clínica. También sería de especial relevancia que tales instrumentos fueran breves, dadas las dificultades que entraña en algunos contextos el empleo de protocolos que consumen excesivo tiempo.

Hasta hace pocos años la evaluación de la sensibilidad a la ansiedad se ha venido llevando a cabo básicamente a través del Anxiety Sensitivity Index (ASI; Reisss, Peterson, Gursky, y McNally, 1986). Esta escala de autoinforme de 16 items, además de su extensa validación en inglés (véase Taylor, 1999), ha sido validada a diversos idiomas, incluido el español (Sandín, Valiente, Chorot y Santed, 2005) y la versión para niños y adolescentes (Sandín, Chorot, Santed y Valiente, 2002; Sandín,
Chorot, Valiente y Santed, 2002). La ASI incluye items referidos a síntomas y consecuencias de tipo físico, social, y mental, aunque la estructura factorial de la escala referida a estas tres dimensiones no se ha demostrado de forma consistente. Taylor y Cox (1998) publicaron la Anxiety Sensitivity Index-Revised (ASI-R), una versión revisada y ampliada de la ASI, de 36 items. Los autores derivaron de forma racional 6 dimensiones (subescalas) que denominaron: miedo a los síntomas cardiovasculares, miedo a los síntomas respiratorios, miedo a los síntomas gastrointestinales, miedo a las reacciones de ansiedad observables públicamente, miedo a los síntomas disociativos y neurológicos, y miedo al descontrol cognitivo. Aunque la ASI-R es mucho más amplia y completa que su predecesora, los estudios factoriales con muestras de diversos países no han apoyado inequívocamente tales dimensiones de la sensibilidad a la ansiedad (Zvolensky et al., 2003). La insatisfacción con la consistencia factorial de ambas versiones (ASI y ASI-R) motivó la construcción, coordinada internacionalmente por Steven Taylor, de una nueva versión de 18 items denominada Anxiety Sensitivity Index -3 (ASI-3; Taylor et al., 2007; Sandín, Valiente, Chorot, y Santed, 2007). La ASI-3 es en esencia una forma depurada y equilibrada de la ASI-R (evalúa de forma consistente las tres dimensiones de sensibilidad a la ansiedad, i.e., las dimensiones física, mental, y social). Actualmente es el instrumento de elección para la evaluación de la sensibilidad a la ansiedad.

Los instrumentos de autoinforme diseñados para la evaluación de los dos restantes constructos del modelo tripartito, esto es, las interpretaciones catastrofistas y la autoeficacia ante el pánico, han seguido un curso más irregular, y han experimentado niveles de validación menos consistentes. En lo que concierne a las interpretaciones catastrofistas de las sensaciones corporales, el instrumento más utilizado ha sido el Body Sensations Interpretation Questionnaire (BSQ; Clark et al., 1997), el cual constituye una versión modificada del Interpretation Questionaire de McNally y Foa (1987). Consiste en 27 sucesos ambiguos, que se presentan cada uno en una página separada, y a los que el individuo debe contestar de acuerdo con tres posibles interpretaciones (unas interpretaciones son negativas, otras positivas, y otras neutrales). Un problema de este instrumento es que, al presentarse en forma de folleto, complica su aplicación tanto en los contextos clínicos como de investigación. Otros instrumentos de autoinforme más convencionales son el Catastrophic Cognitions Questionnaire-Modified (CCQ-M; Khawaja, Oei, y Baglioni, 1994), el Panic Appraisal Inventory - Panic Consequences (PAI-Consecuencias; Telch, Brouillard, Telch, Agras, y Taylor, 1989), y el Panic Belief Inventory (PBI; Wenzel, Sharp, 
Brown, Greenberg, y Beck, 2006). Un problema común al CCQ-M y al PBI consiste en que resultan excesivamente largos para ser utilizados de forma rutinaria (el primero consta de 21 items y el segundo de 35 items). Por otra parte, ambos cuestionarios presentan algunos problemas conceptuales que limitan su capacidad para evaluar las interpretaciones catastrofistas ante los síntomas del ataque de pánico. Así por ejemplo, el PBI evalúa 5 dimensiones heterogéneas y escasamente validadas referidas, respectivamente, a ansiedad anticipatoria y afrontamiento, catástrofes físicas y emocionales, catástrofes emocionales y consecuencias para la salud del ataque de pánico, y auto-desprecio. Así mismo, el CCQ-M, aunque es un cuestionario fácil y rápido de aplicar, más que evaluar interpretaciones catastrofistas asociadas al ataque de pánico evalúa sensibilidad a la ansiedad (p.ej., evalúa la peligrosidad percibida ante síntomas como tener un accidente, sensaciones de temblor, estar enfermo, estar cabreado, no poder relajarse, etc.). El PAI-Consecuencias es una escala de autoinforme de 15 items que evalúan catástrofes de tipo físico, mental (pérdida de control) y social asociadas a los ataques de pánico o a situaciones de mucha ansiedad. Esta escala, por tanto, podría ser un instrumento de elección para evaluar las interpretaciones catastrofistas relacionadas con el ataque de pánico. No obstante, un problema consiste en que algunos items de la escala de la dimensión mental no poseen una clara correspondencia conceptual con tal dimensión; por ejemplo, el ítem "puedo gritar" no está claro que corresponda a una catástrofe mental, del mismo modo que el ítem "puedo llegar a estar completamente histérico" es un ítem demasiado genérico; conceptualmente, ambos items parecen relacionarse más específicamente con la dimensión social que con la mental. Finalmente, varios items se solapan directamente con síntomas del ataque de pánico, tales como "puedo volverme loco" o "puedo morirme".

La evaluación de la autoeficacia ante el pánico se ha llevado a cabo, en general, mediante la utilización de cuestionarios que tienen en común el hecho de evaluar la confianza para afrontar el ataque de pánico en sus diferentes facetas o momentos, tales como al iniciarse los síntomas del ataque, en relación con la situación, asociado a los pensamientos catastrofistas que puedan surgir, etc. Por ejemplo, el cuestionario Panic Attack Self-Efficacy Form (PASE-F; Taylor y Arnow, 1988) evalúa 5 formas de autoeficacia asociada a 5 componentes del pánico (indicadores del inicio del ataque de pánico, ataque de pánico propiamente dicho, sensaciones somáticas, pensamientos de muerte, y urgencia de escapar de la situación). No obstante, no existen datos que apoyen la existencia de estas cinco formas separadas de autoefica- cia ante el pánico, por lo que su utilización debe hacerse con cautela. El Self-Efficacy to Control a Panic Attack Questionnaire (SE-CPAQ; Gauthier, Bouchard, Coté, Laberge, y French, 1993) evalúa, mediante 25 items, la confianza o certeza para controlar un ataque de pánico en relación a tres facetas (escalas) separadas relacionadas con el mismo, i.e., pensamientos (p.ej., "voy a volverme loco"), síntomas (p.ej., "palpitaciones") y situaciones (p.ej., "supermercados"). Estas tres subescalas se circunscriben, respectivamente, a los items de los tres cuestionarios clásicos del grupo de Chambless, esto es, el Agoraphobic Cognitions Cuestionnaire (Chambless, Caputo, Bright, y Gallagher, 1984), el Body Sensation Questionnaire (Chambless et al., 1984) y el Mobility Inventory (Chambless, Caputo, Jasin, Gracely, y Williams, 1985), lo cual, aparte del elevado número de items, resta interés actual al cuestionario. Finalmente, merece la pena resaltar el Panic Appraisal Inventory-Panic Coping (PAI- Coping; Telch et al., 1989), escala del PAI referida al afrontamiento del ataque de pánico. Específicamente, evalúa la creencia o confianza que tiene el paciente para controlar el ataque de pánico. El PAI-Coping consiste en 15 items y proporciona una puntuación total de autoeficacia para afrontar el pánico. Aunque algunos items de esta escala podrían solaparse con otros constructos (p.ej., evitación) y podrían referirse más al afrontamiento que a la autoeficacia propiamente dicha, es una de las escalas más utilizadas actualmente para evaluar la autoeficacia ante pánico.

Como puede apreciarse a partir de lo indicado arriba, actualmente existen instrumentos de autoinforme disponibles para la evaluación de los tres constructos centrales del modelo cognitivo tripartito del TP. No obstante, mientras que existe actualmente un instrumento breve aceptado internacionalmente para la evaluación de la sensibilidad a la ansiedad (i.e., la ASI-3), las medidas referidas a la evaluación de las interpretaciones catastrofistas y la autoeficacia hacia el pánico suelen ser instrumentos relativamente largos y/o con problemas de validez de contenido. Esto sugiere la necesidad de elaborar nuevos instrumentos de autoinforme que posibiliten evaluar estos constructos de forma rápida, válida y fiable. La finalidad del presente estudio ha consistido en examinar la estructura, consistencia interna, fiabilidad y validez de las tres escalas que constituyen el Cuestionario Abreviado del Trastorno de Pánico (CATP). Este cuestionario es una forma abreviada del Cuestionario de Pánico y Agorafobia (CPA; Sandín, Chorot, Valiente, Sánchez-Arribas, y Santed, 2004a). El CATP, mediante 24 items, proporciona tres escalas de autoinforme separadas: (1) Interpretaciones Catastrofistas (EICP;), (2) Escala de Autoeficacia ante el Pánico (EAP), y (3) Escala 
de Gravedad del Pánico (EGP). Adicionalmente pretendíamos obtener información preliminar sobre la validez de la adaptación española de la Panic Disorder Severity Scale-Self Report (PDSS-SR; Houck, Spiegel, Shear, y Rucci, 2002; Sandín, 2002), una versión de autoinforme de la DPSS. De acuerdo con nuestras hipótesis, esperamos obtener evidencia de (a) una estructura trifactorial para la EICP consistente con las tres dimensiones de interpretaciones catastrofistas al pánico, i.e., física, mental y social, (b) una estructura unifactorial para la EAP indicativa de la percepción de autoeficacia ante el pánico, y (c) una estructura unifactorial para las escalas EGP y PDSS-SR, indicativas de un constructo unitario de la gravedad del TP. Así mismo, el estudio pretende proporcionar información relacionada con la fiabilidad y validez convergente y discriminante asociadas a dichas escalas.

\section{Método}

\section{Participantes}

La muestra consistió en 61 participantes con diagnóstico de TP (como diagnóstico principal), con o sin agorafobia, que acudían por primera vez a consulta de salud mental en el Hospital San Juan de la Cruz de Úbeda (Jaén). Los participantes fueron excluidos del estudio si estaban bajo algún tipo de tratamiento psicológico y si tenían menos de 18 años, o algún trastorno neuropsicológico, psicótico, bipolar, o de adicción a sustancias. Los que estaban bajo tratamiento médico (39\%) debían cumplir el requisito de haber seguido una dosis estable durante al menos tres meses antes participar en el estudio. Dieciocho participantes (29.5\%) eran varones y 43 $(70.5 \%)$ eran mujeres. El rango de edad de los participantes era de $18-62$ años $(M=36.8, D T=11.7)$, no existiendo diferencias significativas en edad entre varones $(M=39.4, D T=15.5)$ y mujeres $(M=35.7, D T=9.7)$. Treinta y nueve de los pacientes (64\%) tenían diagnóstico de agorafobia, y 27 (44\%) presentaban uno o más trastornos comórbidos (principalmente otros trastornos de ansiedad).

\section{Instrumentos}

Entrevista para el Diagnóstico de los Trastornos de Ansiedad (ADIS-M; Valiente, Sandín, y Chorot, 2003). La ADIS-M es una entrevista clínica estructurada diseñada para la evaluación y diagnóstico de los trastornos de ansiedad y otros trastornos emocionales, según los criterios del DSM-IV (APA, 1994). La entrevista ha demostrado ser un instrumento fiable y estable temporal- mente para el diagnóstico de los trastornos de ansiedad, incluido el TP (Burgos, 2013; Sandín et al., 2004a; Sandín, Rodero, Santed, y García-Campayo, 2006; Sandín et al., 2015).

Cuestionario Abreviado del Trastorno de Pánico (CATP; Anexo I). Es una forma abreviada del CPA (Sandín et al., 2004b) y se ajusta a los criterios del DSM-5 (APA, 2013) para el diagnóstico del TP. Consiste en 24 items, más un ítem adicional (Ítem 1 del CATP) para registrar la prevalencia anual del ataque de pánico. El CATP incluye las tres escalas siguientes:

(1) Escala de Interpretaciones Catastrofistas al Pánico (EICP; rango 0-48). Es una escala de 12 items (Ítem 5 del CATP) que evalúa la frecuencia de cogniciones catastrofistas asociadas al ataque de pánico, variando la estimación de cada ítem entre 0 ("nunca") y 4 ("siempre"). Los items describen creencias catastrofistas comunes relacionadas con los ataques de pánico, e incluyen pensamientos relacionados con catástrofes de tipo físico (p.ej., tener un ataque cardiaco), mental (p.ej., no poder pensar o razonar), y social (p.ej., llamar atención o hacer el ridículo). Incluye 3 subescalas (4 items cada una) que permiten evaluar de forma separada estas 3 dimensiones (rango de cada subescala o dimensión = 0-16). La EICP se construyó a partir de informes de los pacientes con diagnóstico de TP (Sandín et al., 2004a), y de la evidencia obtenida con otras escalas y estudios sobre el tema (Khawaja et al., 1994; Telch et al., 1989; Westling y Öst, 1993). La consistencia interna de la escala es elevada, tanto para la escala total $(a=.89)$ como para sus tres subescalas (física, $\mathrm{a}=.84$; mental, $\mathrm{a}=.85$; social, $\mathrm{a}=.75)$; también se han publicado datos a favor de la estabilidad temporal ( $r$ test-retest, un mes): .84 (total), .70 (física), .84 (mental), y .74 (social) (Sandín et al., 2015). En el presente estudio hemos obtenido los siguientes coeficientes a: .80 (total), .72 (física), .84 (mental), y .87 (social).

(2) Escala de Autoeficacia ante el Pánico (EAP; rango $=0-16$ ). Es una escala de 4 items (Items 6-9 del CATP) mediante los cuales el individuo estima su capacidad para controlar los ataques de pánico, pudiendo variar entre 0 ("nada") y 4 ("totalmente"). Se basa en escalas construidas previamente (Gauthier et al., 1993; Taylor y Arnow, 1988; Telch et al., 1989) y en el concepto de autoeficacia ante el pánico (Casey et al., 2004a). La escala evalúa la confianza para controlar el ataque de pánico sobre la base de cuatro situaciones relacionadas con: (a) al inicio del ataque de pánico, (b) durante el ataque de pánico, (c) para prevenir el escape en situaciones agorafóbicas, y (d) para manejar las interpretaciones catastrofistas. En estudios previos con la escala de autoeficacia hemos referido niveles excelentes de consistencia interna $(\mathrm{a}=.87)$ y estabili- 
dad temporal $(r$ test-retest, un mes $=.80)$ (Sandín et al., 2015). En el presente estudio, el valor del coeficiente a fue .93).

(3) Escala de Gravedad del Pánico (EGP; rango = 0-32). La EGP consiste en una escala de 8 items (Items 2-4 y 10-14 del CATP), valorados de 0 ("nada") a 4 ("muchísimo"). Incluye los siguientes items: frecuencia de ataques de pánico (último mes), intensidad de los ataques de pánico (media de la subescala de 13 items representativos de los síntomas del ataque de pánico; la subescala incluye dos items adicionales que no se computan para calcular la intensidad), gravedad de los ataques de pánico, preocupación por nuevos ataques o sus consecuencias, cambios desadaptativos en la conducta relacionados con los ataques (p.ej., evitar hacer ejercicio o visitar ciertos lugares), e interferencia laboral, social y familiar. Si únicamente se pretende una información rápida sobre la gravedad del TP, puede prescindirse del ítem de intensidad (Ítem 3 del CATP), ya que la EGP incluye un ítem sobre gravedad percibida de los ataques de pánico (Ítem 4 del CATP; la correlación entre ambas formas de la escala fue de .99). Se ha demostrado tanto la fiabilidad (coeficiente alfa y test-retest un mes) de la escala (.76 y .72, respectivamente), como su validez convergente con gravedad del TP a través de la entrevista clínica ADIS-M $(r=.67)$ (Sandín et al., 2015). En el presente estudio, el coeficiente alfa de la escala fue de $.88(\mathrm{a}=.87 \mathrm{si}$ se suprime el ítem de intensidad). La escala incluye 3 items adicionales para evaluar la frecuencia anual de ataques de pánico, y el nivel general de miedo y molestia durante el ataque de pánico.

Panic Disorder Severity Scale-Self-Report (PDSSSR; Houck et al., 2002). Es una versión de autoinforme de la clásica Panic Disorder Severity Scale (Shear et al. (1997), una escala de estimación clínica. Aplicamos la versión española adaptada por Sandín (2010). Consiste en 7 items, estimados según una escala ordinal de gravedad de 0 a 4 (rango de puntuación total $=0-28$ ). Los items se refieren únicamente a la última semana y evalúan la frecuencia de los ataques de pánico, el nivel de malestar asociado a los ataques de pánico, preocupación o nerviosismo por nuevos ataques, evitación exteroceptiva, evitación interoceptiva, interferencia laboral y familiar, e interferencia social. La PDSS-SR correlacionó .82 con la EGP (Chorot, Sánchez-Arribas, Valiente y Sandín, 2014). En el presente estudio, el coeficiente alfa de la escala fue de .93 .

Panic Appraisal Inventory (PAI; Telch et al., 1989). El PAI incluye tres escalas de 15 items cada una para evaluar, respectivamente, la anticipación del pánico (PAI-Anticipación), las interpretaciones catastrofistas (PAI-Consecuencias), y la autoeficacia ante el pánico
(PAI-Coping). La PAI-Consecuencias posee tres subescalas (5 items cada una) referidas, respectivamente a los tres tipos de catástrofes asociadas a la evaluación del ataque de pánico (i.e., consecuencias físicas, sociales, y pérdida de control). Los items se responden según una escala graduada de cero a 10 puntos (rango de la escala total $=0-150$; rango de cada subescala $=0-50)$; el participante debe contestar indicando hasta qué punto le perturba o preocupa lo indicado en cada uno de una lista de 15 pensamientos. La PAI-Coping consiste en 15 items evaluados según una escala de 0 a 10 puntos $($ rango $=$ 0-150); el individuo contesta indicando hasta qué punto se siente seguro o confía en que puede superar o experimentar una serie de situaciones relacionadas con el pánico (p.ej., "experimentar un ataque de pánico sin escapar de la situación"). En relación con el actual trabajo únicamente se han utilizado las dos últimas escalas. La traducción al español de la PAI se llevó a cabo por los autores del artículo, siendo coordinada por el último autor (BS). La traducción inversa fue realizada por un psicólogo clínico con idioma nativo inglés. En el presente estudio hemos obtenido los siguientes coeficientes a: .92 (PAI-Consecuencias), .85 (físico), .86 (social) y .82 (pérdida de control), y .93 (PAI-Coping).

\section{Procedimiento}

Los participantes fueron evaluados tras acudir al centro clínico en busca de atención psicológica, y antes de su inclusión en un programa de tratamiento cognitivo-conductual. Fueron seleccionados los que cumplían el diagnóstico de TP (con o sin agorafobia) como diagnóstico principal, siguiendo los criterios del DSM-IV. Inicialmente los pacientes fueron preseleccionados por facultativos del hospital (psicólogos o psiquiatras). En una segunda fase, tras haber sido obtenido el consentimiento informado, los participantes fueron entrevistados de nuevo por el primer autor aplicando la entrevista clínica ADIS-M. La tasa de acuerdo con el diagnóstico inicial para el diagnóstico del TP fue del 100\%. Posteriormente, los participantes cumplimentaron en una sesión el protocolo de evaluación con las pruebas de autoinforme descritas arriba.

\section{Tratamiento estadístico de los datos}

Se aplicaron análisis factoriales separados para cada una de las 4 escalas evaluadas (las tres escalas del CATP y la PDSS-SR). Antes de efectuarse los análisis se comprobó que la matriz de correlaciones era adecuada para los mismos; tanto el estadístico de Bartlett $(\geq .72)$ como el test de Kaiser-Meyer-Olkin $(p<.001)$ indicaron que la 
matriz resultaba adecuada en todos los análisis factoriales llevados a cabo. Para la obtención de los factores aplicamos el método de mínimos cuadrados no ponderados (ULS) basado en las correlaciones policóricas. La determinación del número de factores a extraer se efectuó aplicando el método de análisis paralelo de implementación óptima (Timmerman y Lorenzo-Seva, 2011). Además de los análisis factoriales, calculamos los estadísticos descriptivos y análisis correlacionales (correlación de Pearson) para examinar la validez convergente y discriminante. En lo que concierne a los análisis descriptivos, aparte de las puntuaciones calculadas sumando las estimaciones directas en cada ítem, calculamos las puntuaciones transformadas (ajustadas), esto es, puntuaciones ajustadas al rango de estimación de los items (esta forma de presentación facilita su interpretación). La transformación consistió en dividir la puntuación obtenida en la escala por el número de items de la escala. Todos los análisis estadísticos se llevaron a cabo utilizando los programas SPSS.19, y FACTOR 8.1 (Lorenzo-Seva y Ferrando, 2006).

\section{Resultados}

El proceso de presentación de los resultados se llevará a cabo en dos fases y referidos de forma separada a las tres escalas del CATP y a la PDSS-SR. En primer lugar examinamos la estructura factorial de las escalas y la fiabilidad (coeficientes alfa y correlaciones ítem-escala/ subescala). En segundo lugar presentaremos los descriptivos (medias y desviaciones típicas) y las correlaciones entre todas las variables del estudio con objeto de proporcionar información sobre la validez convergente y discriminante de las escalas.

\section{Estructura factorial y fiabilidad de las escalas EICP, EAP, EGP y PDSS-SR}

El análisis factorial de la escala de interpretaciones catastrofistas (EICP), y basándonos en el criterio del análisis paralelo, sugirió una estructura de tres factores que representan, respectivamente, las dimensiones social, física y mental (véase la Tabla 1). Los autovalores correspondientes a las cuatro primeras variables fueron $3.77,1.95,1.85$ y .73 ; los tres factores extraídos explicaban el $64 \%$ de la varianza total. El ajuste del modelo trifactorial a los datos fue excelente [goodness of fit index $(\mathrm{GFI})=.99$, root mean square of residuals $($ RMSR $)=.046]$. En la Tabla 1 exponemos las saturaciones referidas únicamente al factor relevante (los pesos factoriales de los items de la escala en los factores no relevantes oscilaron entre .01 y .22). Como puede apreciarse por el valor de los pesos factoriales y por la ausencia de saturaciones en los factores irrelevantes, la estructura factorial de la EICP es robusta y consistente, y sugiere que los tres factores representan las tres dimensiones sugeridas en la literatura de las interpretaciones catastrofistas a los síntomas del pánico (i.e., fí-

Tabla 1. Análisis factorial de la Escala de Interpretaciones Catastrofistas al Pánico (EICP) basado en las correlaciones policóricas.

Método de mínimos cuadrados no ponderados (ULS) y análisis paralelo para la extracción de los factores, y rotación promax normalizada. Se indican los pesos factoriales para cada factor relevante, la comunalidad $\left(h^{2}\right)$, los coeficientes alfa de Cronbach y las correlaciones corregidas ítem-subescala $\left(r_{\text {is }}\right)$

\begin{tabular}{|c|c|c|c|}
\hline Ítem EICP & Peso factorial & $h^{2}$ & $r_{\text {is }}$ \\
\hline \multicolumn{4}{|l|}{ Factor 1. Social $(\alpha=0.87)$} \\
\hline 3. La gente se fijará o reirá de mí & .88 & .75 & .81 \\
\hline 6. Será una situación embarazosa & .66 & .53 & .82 \\
\hline 9. Puedo llamar la atención o hacer el ridículo & .65 & .52 & .83 \\
\hline 11. Puedo hacer una escena delante de los demás & .67 & .43 & .87 \\
\hline \multicolumn{4}{|l|}{ Factor 2. Físico $(\alpha=0.72)$} \\
\hline 1. Puedo tener un ataque al corazón & .62 & .46 & .74 \\
\hline 2. Podré desmayarme & .39 & .24 & .75 \\
\hline 8. Puedo tener una trombosis o un derrame cerebral & .71 & .51 & .56 \\
\hline 12. Podré asfixiarme & .85 & .70 & .56 \\
\hline \multicolumn{4}{|l|}{ Factor 3. Mental $(\alpha=0.84)$} \\
\hline 4. Mi mente no funcionará bien & .61 & .39 & .83 \\
\hline 5. Puedo perder el control de mis sentidos & .71 & .63 & .78 \\
\hline 7. No podré pensar o razonar & .72 & .53 & .79 \\
\hline 10. Me desequilibraré mentalmente & .79 & .59 & .77 \\
\hline
\end{tabular}


Tabla 2. Análisis factorial de la Escala de Autoeficacia ante el Pánico (EAP) basado en las correlaciones policóricas. Método de mínimos cuadrados no ponderados (ULS) y análisis paralelo para la extracción de los factores. Se indican los pesos factoriales, la comunalidad $\left(h^{2}\right)$, el coeficiente alfa de Cronbach y las correlaciones corregidas ítem-escala $\left(r_{\text {is }}\right)$

\begin{tabular}{lccc}
\hline \multicolumn{1}{c}{ Ítem EAP } & Peso factorial & $h^{2}$ & $r_{\text {is }}$ \\
\hline 1. Ante pensamientos catastrofistas & .82 & .67 & .84 \\
2. Ante las primeras señales del ataque de pánico & .76 & .58 & .75 \\
3. Cuando está experimentando los síntomas del ataque de pánico & .83 & .69 & .88 \\
4. Cuando experimenta síntomas de la crisis en algún lugar agorafóbico & .82 & .67 & .90 \\
Coeficiente alfa de Cronbach $=.93$ & & \\
\hline
\end{tabular}

Tabla 3. Análisis factorial de la Escala de Gravedad del Pánico (EGP) basado en las correlaciones policóricas. Método de mínimos cuadrados no ponderados (ULS) y análisis paralelo para la extracción de los factores. Se indican los pesos factoriales, la comunalidad $\left(h^{2}\right)$, el coeficiente alfa de Cronbach y las correlaciones corregidas ítem-escala $\left(r_{\text {is }}\right)$

\begin{tabular}{lrrr}
\hline \multicolumn{1}{c}{ Ítem EGP } & Peso factorial & \multicolumn{1}{c}{$h^{2}$} & $r_{\text {is }}$ \\
\hline 1. Frecuencia ataques de pánico (último mes) & .45 & .20 & .33 \\
2. Intensidad de los ataques de pánico & .66 & .44 & .63 \\
3. Gravedad de los ataques de pánico & .86 & .74 & .77 \\
4. Preocupación por nuevos ataques de pánico o por sus consecuencias & .67 & .45 & .58 \\
5. Cambios comportamentales relacionados con los ataques de pánico & .76 & .57 \\
6. Interferencia laboral debida a los ataques de pánico & .80 & .65 & .72 \\
7. Interferencia social debida a los ataques de pánico & .86 & .74 \\
8. Interferencia familiar debida a los ataques de pánico & .79 & .63 \\
Coeficiente alfa de Cronbach $=.88$ & & .71 \\
\hline
\end{tabular}

sica, social y mental). Los valores obtenidos para la fiabilidad (alfa de Cronbach) sugieren que las tres subescalas poseen una adecuada consistencia interna. Así mismo, las correlaciones corregidas ítem-subescala son elevadas para todos los items de la escala (rango $=.56-.87$ ).

Un procedimiento de análisis factorial similar se aplicó al resto de las escalas. En lo que concierne a los items de la escala de autoeficacia (EAP), el análisis paralelo sugirió un único factor, el cual representa el constructo de autoeficacia ante el pánico (véase la Tabla 2). Los tres primeros autovalores fueron como sigue: $2.95, .41$ y .33 ; el factor explicaba el $74 \%$ de la varianza total. Los datos sobre la bondad de ajuste del modelo unifactorial fueron excelentes $(\mathrm{GFI}=1.00$, RMSR $=.007)$. Así mismo, las elevadas saturaciones observadas en el factor apoyan su elevada consistencia interna del mismo (rango de las saturaciones $=.76-$ $.83)$, y sugieren que los cuatro items de la AEP describen un único factor de autoeficacia ante el pánico. Tanto el coeficiente alfa de Cronbach como las correlaciones corregidas ítem-escala sugieren una excelente consistencia interna de la escala.

Los resultados obtenidos en relación con la escala de gravedad del trastorno de pánico (EGP) se indican en la Tabla 3. Basándonos en el análisis paralelo se obtuvo un factor que explicaba el $60 \%$ de la varianza total. Las puntuaciones de los tres primeros autovalores fueron $4.80, .89$ y .66 . Los índices de bondad de ajuste del modelo fueron GFI $=.99$ y RMSR $=.063$. Los niveles de las saturaciones de las variables en el factor oscilaron entre .45 y .86, sugiriendo una estructura factorial consistente y bastante robusta. El factor representa claramente una dimensión de gravedad del TP. Los valores más bajos corresponden al ítem que evalúa la frecuencia de ataques de pánico experimentados en el último mes (denota la percepción de ataques de pánico ocurridos, pero es una variable menos subjetiva que el resto de variables del factor). El coeficiente alfa de Cronbach denota un nivel excelente de consistencia interna. Las correlaciones corregidas ítem-escala son todas elevadas, excepto la referida al ítem de frecuencia de ataques de pánico que es moderada.

Finalmente, aplicamos un mismo procedimiento de análisis de los datos para la escala Panic Disorder Severity Scale-Self-Report (PDSS-SR). Los resultados basados en el análisis paralelo indican que se trata de una escala claramente unidimensional (véase la Tabla 4). El factor explicaba el $70 \%$ de la varianza total, siendo las puntuaciones de los tres primeros autovalores como sigue: $4.89, .64$, y .41. Los índices de bondad de ajuste del modelo fueron: GFI $=1.0, \mathrm{RMSR}=.052$. 
Tabla 4. Análisis factorial de la Panic Disorder Severity Scale-Self Report (PDSS-SR) basado en las correlaciones policóricas. Método de mínimos cuadrados no ponderados (ULS) y análisis paralelo para la extracción de los factores. Se indican los pesos factoriales, la comunalidad $\left(h^{2}\right)$, el coeficiente alfa de Cronbach, y las correlaciones corregidas ítem-escala $\left(r_{\text {is }}\right)$

\begin{tabular}{lrrr}
\hline \multicolumn{1}{c}{ Ítem PDSS-SR } & Peso factorial & $h^{2}$ & $r_{\text {is }}$ \\
\hline 1. Frecuencia ataques de pánico (última semana) & .74 & .54 & .69 \\
2. Malestar de los ataques de pánico & .81 & .65 & .82 \\
3. Preocupación por nuevos ataques de pánico o por temores relacionados & .81 & .65 & .84 \\
4. Evitación de situaciones agorafóbicas & .76 & .58 & .74 \\
5. Evitación de actividades que producen sensaciones físicas similares al pánico & .84 & .70 \\
6. Interferencia laboral o familiar debida al pánico & .84 & .71 \\
7. Interferencia social debida al pánico & .83 & .84 \\
Coeficiente alfa de Cronbach = .93 & & .84 \\
\hline
\end{tabular}

Las saturaciones de cada ítem en el factor eran todas elevadas, variando entre .74 y .84 , lo que sugiere una estructura unidimensional muy consistente. Así mismo, la elevada consistencia interna de la escala quedó demostrada, tanto por el elevado coeficiente alfa de Cronbach, como a través de las correlaciones corregidas ítem-escala.

\section{Estadísticos descriptivos y correlaciones entre las variables}

En la Tabla 5 presentamos las medias y desviaciones típicas correspondientes a las variables del estudio. No encontramos diferencias estadísticamente significativas en función del sexo para ninguna de las variables. Los resultados de las comparaciones entre medias (varones vs. mujeres) reflejaron valores de $t$ que variaban entre .19 (PAI Consecuencias total; $p>.05$ ) y 1.15 (EICP-Físico; $p>.05)$. Según reflejan las puntuaciones medias obtenidas en las tres subescalas de la EICP, puede observarse que la media en la dimensión social tiende a ser inferior a la de las otras dos dimensiones de interpretaciones catastrofistas; aunque tal fenómeno no se observa en relación con las subescalas de interpretaciones catastrofistas del PAI.

Si nos basamos en las medias ajustadas, se evidencia que los valores medios de gravedad del TP se distribuyen entre los niveles de bastante y mucha gravedad en ambas

Tabla 5. Medias, desviaciones típicas e intercorrelaciones para todas las variables del estudio

\begin{tabular}{|c|c|c|c|c|c|c|c|c|c|c|c|c|}
\hline Variable & 1 & & 3 & 4 & 5 & 6 & 7 & 8 & 9 & 10 & 11 & 12 \\
\hline 1. EGP & - & & & & & & & & & & & \\
\hline 2. PDSS-SR & .81 & - & & & & & & & & & & \\
\hline 3. EICP Total & .75 & .54 & - & & & & & & & & & \\
\hline 4. EICP Física & .63 & .48 & .64 & - & & & & & & & & \\
\hline 5. EICP Mental & .63 & .48 & .70 & .18 & - & & & & & & & \\
\hline 6. EICP Social & .32 & .18 & .73 & .17 & .31 & - & & & & & & \\
\hline 7. PAI Consecuencias total & .77 & .56 & .81 & .64 & .57 & .44 & - & & & & & \\
\hline 8. PAI Consecuencias físicas & .64 & .48 & .57 & .75 & .30 & .18 & .86 & - & & & & \\
\hline 9. PAI Consecuencias mentales & .71 & .55 & .68 & .45 & .66 & .32 & .89 & .62 & - & & & \\
\hline 10. PAI Consecuencias sociales & .70 & .46 & .83 & .49 & .57 & .66 & .89 & .63 & .73 & - & & \\
\hline 11. EAP & -.43 & -.47 & -.38 & -.25 & -.29 & -.28 & -.30 & -.20 & -.28 & -.30 & - & \\
\hline 12. PAI Coping & -.65 & -.58 & -.59 & -.52 & -.45 & -.27 & -.57 & -.51 & -.48 & -.52 & .66 & - \\
\hline Media & 22.20 & 16.03 & 27.70 & 9.73 & 10.40 & 7.55 & 81.57 & 30.31 & 25.50 & 25.75 & 4.75 & 55.85 \\
\hline$D T$ & 6.04 & 8.17 & 7.62 & 3.59 & 3.53 & 3.86 & 33.67 & 13.15 & 12.32 & 12.65 & 2.97 & 30.15 \\
\hline Media ajustada* & 2.77 & 2.29 & 2.30 & 2.43 & 2.60 & 1.88 & 5.43 & 6.06 & 5.10 & 5.15 & 1.19 & 3.72 \\
\hline$D T$ ajustada & .75 & 1.16 & .63 & .89 & .88 & .96 & 2.23 & 2.63 & 2.46 & 2.53 & .74 & 2.01 \\
\hline
\end{tabular}

Nota . EAP = Escala de Autoeficacia ante el Pánico, EGP = Escala de Gravedad del Pánico, EICP = Escala de Interpretaciones Catastrofistas al Pánico, PAI = Panic Appraisal Inventory, PDSS-SR = Panic Disorder Severity Scale—Self Report. Correlaciones $\geq .33, p<.01 ;$ correlaciones $\geq .25, p<.05$. $*$ Rango posible (media ajustada) para las variables 7, 8, 9, 10 y $12=0-10$; para el resto de variables $=0-4$ (véase el epígrafe sobre tratamiento estadístico de los datos). En negrita se indican las correlaciones indicativas de validez convergente para las escalas del CATP. 
escalas de gravedad del pánico (EGP y PDSS-SR). Así mismo, las puntuaciones medias en interpretaciones catastrofistas (EICP) de tipo físico y mental indican que estás ocurrían con bastante frecuencia durante las experiencias de los ataques de pánico (se situaban entre varias veces y casi siempre), siendo menos frecuentes las interpretaciones de tipo social. Finalmente, podemos observar que la media en la EAP es de 1.19, lo cual indica que los pacientes confiaban poco en su capacidad para controlar los ataques de pánico. Algo similar se desprende a partir de la media obtenida en la escala PAI-Coping.

Las correlaciones entre todas las variables utilizadas en la investigación se indican en la Tabla 5. En primer lugar, podemos apreciar que las tres escalas del CATP (y sus correspondientes variables) presentan niveles adecuados de validez convergente, de acuerdo con las elevadas correlaciones obtenidas con constructos similares evaluados a través de otros instrumentos. Así, observamos que la gravedad del TP evaluada mediante la EGP correlaciona .81 con la gravedad del TP evaluada a través de la PDSS-SR. De forma similar, las subescalas de la EICP correlacionan de forma elevada con las correspondientes escalas del PAI-Consecuencias (rango .66.75), así como también las puntuaciones totales en interpretaciones catastrofistas (correlación entre EICP y PAI-Consecuencias $=.81$ ). La escala de autoeficacia ante el pánico (EAP) también correlaciona de forma elevada con la correspondiente escala del PAI (i.e., la PAI-Coping). En contraste, el análisis correlacional indica evidencia de validez discriminante, constatado a través de las bajas o moderadas correlaciones entre constructos separados (p.ej., las correlaciones entre subescalas no relevantes de interpretaciones catastrofistas variaron entre .18 y .57 ).

También podemos observar correlaciones elevadas y significativas entre las diferentes variables de interpretaciones catastrofistas y las variables de gravedad del TP (EGP y DPSS-SR). En este sentido, cabe subrayar que la subescala social de la EICP correlaciona de forma más baja que el resto de subescalas (física y mental), no siendo estadísticamente significativa su correlación con la DPSS-SR. En contraste, como cabría esperar, ambas variables de autoeficacia (EAP y PAI-Coping) correlacionan de forma negativa y significativa con la gravedad del TP (en ambas variables de gravedad).

\section{Discusión}

El objetivo principal del presente estudio ha consistido en examinar las propiedades psicométricas del CATP, un cuestionario breve que permite evaluar de forma rápida tres constructos clínicos esenciales del TP, esto es, las interpretaciones catastrofistas al pánico (EICP), la autoeficacia ante el ataque de pánico (EAP), y la gravedad del TP (EGP). Nuestra hipótesis de partida consistía en que debería evidenciarse una estructura trifactorial para la escala de interpretaciones catastrofistas y una estructura unifactorial para las dos restantes. Así mismo, también habíamos hipotetizado una estructura unifactorial para la escala de gravedad del pánico del grupo de Shear (Houck et al., 2002). Por otra parte, esperábamos correlaciones particularmente elevadas entre las variables del CATP y otros constructos convergentes, y correlaciones moderadas o bajas entre las variables del CATP y otros constructos más alejados, examinando de este modo la validez convergente y divergente de las escalas y subescalas del CATP.

Los resultados obtenidos son en general coherentes con nuestras hipótesis sobre la estructura factorial de las escalas examinadas. Los datos indican que la escala de interpretaciones catastrofistas EICP está definida por tres factores separados que representan las tres dimensiones reconocidas en la literatura de forma consistente sobre las interpretaciones catastrofistas del pánico, esto es, las dimensiones de tipo físico, mental y social (Casey et al., 2004a; Khawaja et al., 1994; Sandín et al., 2015; Telch et al., 1989; Westling y Öst, 1993; Zoellner, Craske y Rapee, 1996). Estos resultados son también consistentes con las tres dimensiones (física, mental y social) que caracterizan el constructo de sensibilidad a la ansiedad (Sandín et al., 2007; Taylor, 1999; Taylor et al., 2007). Los resultados sugieren, así mismo, la posible utilidad clínica de la EICP, así como también para la investigación, ya que nos permite obtener puntuaciones separadas para cada una de las tres dimensiones de cogniciones catastrofistas, aparte de la puntuación total. Tanto la puntuación total, como las puntuaciones separadas han demostrado ser suficientemente fiables (consistencia interna), siendo estos resultados similares a los obtenidos recientemente por nuestro grupo sobre fiabilidad (consistencia interna) y estabilidad temporal de la escala y las tres subescalas (Sandín et al., 2015).

En lo que concierne a las dos restantes escalas del CATP investigadas (EAP y EGP), los resultados indican que se trata de dos constructos unidimensionales. La escala EAP, aunque hace alusión a cuatro tipos de situaciones o aspectos diferentes asociados al ataque de pánico, ha demostrado estar representada por un factor sólido de autoeficacia ante los ataques de pánico. Por tanto, es consistente con la hipótesis mantenida en la literatura sobre la posibilidad de que este tipo de autoeficacia pueda constituir un fenómeno unitario, al menos cuando se refiere al afrontamiento genérico del ataque de pánico (Casey et al., 2004a, 2004b; Fenz et al., 2013; Sandín et 
al., 2015; Telch et al., 1989). Considerando, por otra parte, la elevada consistencia interna de la escala observada a través de los resultados del presente estudio, al igual que la elevada consistencia interna y estabilidad temporal constatada previamente por nuestro grupo (Sandín et al., 2015), podríamos concluir que la EAP puede utilizarse de forma fiable para obtener una puntuación global de la autoeficacia ante los ataques de pánico. Algo similar podría afirmarse con respecto a la EGP, la cual ha demostrado una elevada estabilidad temporal, consistencia interna y factorial representada esta última por un único factor, consistente con los datos previos obtenidos por nuestro grupo basados en una forma de 6 items (Chorot et al., 2014; Sandín et al., 2015).

Adicionalmente, en nuestro estudio hemos examinado la estructura factorial de la PDSS-SR de Houck et al. (2002). La estructura factorial es muy consistente y sugiere un claro y único factor de gravedad del TP. Estos datos contrastan con los resultados referidos recientemente por Santacana et al. (2014) con otra versión española de la PDSS-SR. Estos autores obtuvieron dos factores; un factor estaba configurado por los dos primeros items de la escala, y un segundo factor por los cinco items restantes. Nuestros resultados sobre la fiabilidad de la escala son similares a los obtenidos por Houck et al. (2002) y Santacana et al. (2014). La puntuación media obtenida en nuestro estudio (16.03) es sólo ligeramente superior a la obtenida en nuestro anterior estudio (Sandín, Rodero, Santed y García-Campayo, 2006) con la versión española de la forma de estimación por el clínico (Bulbena y Martín Santos, 1992) (en el estudio de Sandín et al. la media fue de $14.26, D T=4.67$, durante el pre-tratamiento), así como también a la obtenida por Santacana et al. (2014) en la condición de pretratamiento. En contraste, la puntuación total en la forma de autoinforme obtenida por Houck et al. (2002) fue de 9.0 $(D T=6.6)$, lo cual denota una notable diferencia con respecto a las puntuaciones obtenidas con ambas versiones españolas (escala de autoinforme y escala de estimación por el clínico). Tal diferencia podría deberse a que la muestra de Houck et al. se encontraba en su mayoría bajo tratamiento psicológico.

Las correlaciones entre las variables del estudio proporcionan apoyo empírico a la validez convergente para las tres escalas del CATP. En primer lugar, tanto la puntuación total de la EICP como las puntuaciones en las tres subescalas, correlacionan de forma elevada y significativa con las correspondientes variables de la PAI-Consecuencias. Por ejemplo, ambas escalas (puntuaciones totales) correlacionan .81, lo cual indica que en gran medida evalúan el mismo constructo. Algo similar ocurre con las correlaciones entre las subescalas similares (p.ej., la subescala EICP de tipo físico correlaciona .75 con su homóloga física de la PAI-Consecuencias). También proporcionamos apoyo empírico sobre la validez convergente de la CATP-Autoeficacia, tal y como deriva de la elevada correlación con la PAI-Coping. Finalmente, la elevada correlación entre las dos variables de gravedad del TP denota que la EGP evalúa un constructo similar al evaluado por la DPSS-SR.

Es importante destacar la capacidad discriminante de la EICP al asociarse a la gravedad del TP (evaluada ésta por cualquiera de las dos medidas utilizadas en el estudio), capacidad que también se observa para las variables del PAI. Como puede apreciarse (véase la Tabla 5), la escala y subescalas de interpretaciones catastrofistas del CATP correlacionan de forma elevada, positiva y significativa con la gravedad del TP, mientras que la escala de autoeficacia lo hace de forma negativa. Estos datos son consistentes con evidencia reciente sobre la asociación de factores cognitivos positivos (i.e., autoeficacia ante el pánico) y negativos (i.e., interpretaciones catastrofistas al pánico) con la gravedad del TP (Casey et al., 2004b; Sandín et al., 2015), la cual indica, así mismo, un claro apoyo a los modelos tripartito (Sandín et al., 2015) e integrativo (Casey et al., 2004a) del TP. Por otra parte, las correlaciones entre moderadas y bajas de la dimensión social de la EICP con la gravedad del TP está en línea con evidencia reciente que tiende a señalar una menor implicación de esta dimensión en la etiopatogenia del TP, en comparación con las dimensiones física y mental (véase Sandín et al., 2015).

Concluyendo, en el presente estudio hemos proporcionado evidencia relevante y consistente sobre la validación del CATP. Los datos aportados en esta investigación sugieren que las tres escalas que componen este cuestionario son válidas y fiables, y podrían ser de gran utilidad tanto en la investigación como en la aplicación en contextos clínicos relacionados con el trastorno de pánico. Las tres escalas que integran el CATP son muy breves y rápidas en su aplicación, por lo que podrían ser especialmente útiles en este sentido. Así mismo, puesto que las escalas se refieren a aspectos positivos (i.e., autoeficacia) y negativos (i.e., interpretaciones catastrofistas y gravedad del TP), pueden resultar de especial interés para la evaluación de constructos relevantes de los modernos modelos cognitivos del TP (véase Sandín et al., 2015). Por otra parte, proporcionamos evidencia empírica que apoya la fiabilidad y validez de la versión española de la PDSS-SR de Houck et al. (2002). Adicionalmente, considerando que el ataque de pánico constituye un marcador (o especificador) que puede asociarse a múltiples tipos de trastornos mentales (APA, 2013; Sandín, 2013) y actuar en cierto modo como un 
fenómeno transdiagnóstico (véase Belloch, 2012; Sandín, Chorot y Valiente, 2012; Titov, Dear, Johnston y Terides, 2012) implicando la regulación emocional y otros posibles fenómenos cognitivos (Aldao, 2012), las escalas EICP y EAP podrían ser también de utilidad para evaluar las interpretaciones catastrofistas y la autoeficacia al pánico como constructos transdiagnósticos presentes en diversos trastornos mentales, más allá del propio TP.

El presente estudio posee algunas limitaciones que merece la pena señalar. Una primera viene dada por el bajo tamaño de la muestra. No obstante, antes de efectuar los análisis factoriales nos aseguramos de que las matrices de correlaciones eran apropiadas y la distribución era normal (los niveles de curtosis y asimetría eran aceptables para todas las variables estudiadas), aplicando además correlaciones policóricas para minimizar el posible peligro debido a la naturaleza ordinal de las estimaciones. En segundo lugar, el estudio proporciona evidencia preliminar sobre la validación de las cuatro escalas estudiadas. Se precisan nuevos estudios, tanto transversales como longitudinales (empleando también otros tipos de trastornos de ansiedad además del TP), para probar la validez de las mismas en sus diferentes facetas (predictiva, discriminante, etc.), y para determinar los procesos de cambio en estas variables (interpretaciones catastrofistas, autoeficacia y gravedad del TP) asociados a programas de terapia cognitivo-conductual del TP validados empíricamente.

\section{Referencias}

Aldao, A. (2012). Emotion regulation strategies as transdiagnostic processes: a closer look at the invariance of their form and function. Revista de Psicopatología y Psicología Clínica, 17, 261-277.

American Psychiatric Association (APA) (1994). Diagnostic and statistical manual of mental disorders (4th ed.). DSM-IV. Washington, DC: Author.

American Psychiatric Association (APA) (2013). Diagnostic and statistical manual of mental disorders (5th ed.). DSM-5. Washington, DC: Author.

Austin, D. W., \& Richards, J. C. (2006). A test of core assumptions of the catastrophic misinterpretation model of panic disorder. Cognitive Therapy and Research, 30, 53-68.

Belloch, A. (2012). Propuestas para un enfoque transdiagnóstico de los trastornos mentales y del comportamiento: evidencia, utilidad y limitaciones [Proposals for a transdiagnostic perspective of mental and behavioural disorders: Evidence, usefulness, and limitations]. Revista de Psicopatología y Psicología Clínica, 17, 295-311.

Bernstein, A., Zvolensky, M.J., Sandin, B., Chorot, P., \& Stickle, T. (2008). Body vigilance: Bi-national examination of the construct. Depression and Anxiety, 25, E81-E9.
Bouton, M. E., Mineka, S., \& Barlow, D. H. (2001). A modern learning theory perspective on the etiology of panic disorder. Psychological Review, 108, 4-32.

Bulbena, A., y Martín Santos, R. (1992). Escala de Gravedad del Trastorno de Pánico [Panic Disorder Severity Scale]. Departamento de Psiquiatría, Facultad de Medicina. Universidad de Barcelona (policopiado).

Burgos, D. (2013). Sesgos cognitivos atencionales y de interpretación en personas mayores con diagnóstico de trastorno de ansiedad generalizada. Tesis doctoral, Universidad Autónoma de Madrid.

Casey, L. M., Oei, T. P., \& Newcombe, P. A. (2004a). An integrated cognitive model of panic disorder: The role of positive and negative cognitions. Clinical Psychology Review, 24, 529555.

Casey, L. M., Oei, T. P., Newcombe, P. A., \& Kenardy, J. (2004b). The role of catastrophic misinterpretation of bodily sensations and panic self-efficacy in predicting panic severity. Journal of Anxiety Disorders, 18, 325-340.

Chambless, D. L., Caputo, G. C., Bright, P., \& Gallagher, R. (1984). Assessment of fear of fear in agoraphobics: the body sensations questionnaire and the agoraphobic cognitions questionnaire. Journal of Consulting and Clinical Psychology, 52, 1090.

Chambless, D. L., Caputo, G. C., Jasin, S. E., Gracely, E. J., \& Williams, C. (1985). The mobility inventory for agoraphobia. Behaviour Research and Therapy, 23, 35-44.

Chorot, P. (1989). Teoría de incubación de la ansiedad. En B. Sandín y J. Bermúdez (Eds.), Procesos emocionales y salud (pp. 75-110). Madrid: UNED.

Chorot, P. (1991). Teoría de la incubación de la ansiedad: evidencia empírica. Revista de Psicología General y Aplicada, 44, 435-445.

Chorot, P., Sánchez-Arribas, C., Valiente, R. M., y Sandín, B. (2014). Coping with panic: The Panic Self-efficacy Scale (PSES). In Poster session to be presented at the 45th Annual European Association for Behavioural and Cognitive Therapies (August-September 2015), Jerusalem, Israel (Submitted).

Chorot, P., \& Sandín, B. (1993). Effects of UCS intensity and duration of exposure of nonreinforced CS on conditioned electrodermal responses: An experimental analysis of the incubation theory of anxiety. Psychological Reports, 73, 931-941.

Clark, D. M. (1986). A cognitive approach to panic. Behaviour Research and Therapy, 24, 461-470.

Clark, D. M. (1999). Anxiety disorders: Why they persist and how to treat them. Behaviour Research and Therapy, 37, S5-S27.

Clark, D. M., Salkovskis, P. M., Öst, L. G., Breitholtz, E., Koehler, K. A., Westling, B. E., ... \& Gelder, M. (1997). Misinterpretation of body sensations in panic disorder. Journal of Consulting and Clinical Psychology, 65, 203-213.

Craske, M. G., \& Barlow, D. H. (2008). Panic disorder and agoraphobia. In D. H. Barlow (Ed.), Clinical handbook of psychological disorders (4th ed.) (pp. 1-64). New York: The Guilford Press.

Eysenck, H. J. (1985). Incubation theory of fear/anxiety. In S. Reiss and R. R. Bootzin (Eds.), Theoretical issues in behavior therapy (83-105). New York: Academic Press.

Fava, L., \& Morton, J. (2009). Causal modeling of panic disorder theories. Clinical Psychology Review, 29, 623-637. 
Fentz, H. N., Hoffart, A., Jensen, M. B., Arendt, M., O’Toole, M. S., Rosenberg, N. K., \& Hougaard, E. (2013). Mechanisms of change in cognitive behaviour therapy for panic disorder: The role of panic self-efficacy and catastrophic misinterpretations. Behaviour Research and Therapy, 51, 579-587.

Gallagher, M. W., Payne, L. A., White, K. S., Shear, K. M., Woods, S. W., Gorman, J. M., \& Barlow, D. H. (2013). Mechanisms of change in cognitive behavioral therapy for panic disorder: The unique effects of self-efficacy and anxiety sensitivity. Behaviour Research and Therapy, 51, 767-777.

Gauthier, J. G., Bouchard, S., Cote, G., Laberge, B., \& French, D. (1993). Development of two scales measuring self-efficacy to control panic attacks. Canadian Psychology, 30(2a), 305.

Gorman, J. M., Kent, J. M., Sullivan, G. M., \& Coplan, J. D. (2004). Neuroanatomical hypothesis of panic disorder, revised. American Journal of Psychiatry, 157, 493-505.

Houck, P. R., Spiegel, D. A., Shear, M. K., \& Rucci, P. (2002). Reliability of the self-report version of the panic disorder severity scale. Depression and Anxiety, 15, 183-185.

Khawaja, N. G., \& Oei, T. P. (1998). Catastrophic cognitions in panic disorder with and without agoraphobia. Clinical Psychology Review, 18, 341-365.

Khawaja, N. G., Oei, T. P., \& Baglioni Jr, A. J. (1994). Modification of the Catastrophic Cognitions Questionnaire (CCQ-M) for normals and patients: Exploratory and LISREL analyses. Journal of Psychopathology and Behavioral Assessment, 16, 325-342.

Klein, D. F. (1993). Fasse suffocation alarms, spontaneous panic, and related conditions: An integrative hypothesis. Archives of General Psychiatry, 50, 306-317.

Lorenzo-Seva, U., \& Ferrando, P. J. (2006). FACTOR: A computer program to fit the exploratory factor analysis model. Behavior Research Methods, 38, 88-91.

McNally, R. J. (1990). Psychological approaches to panic disorder: A review. Psychological Bulletin, 108, 403.

McNally, R. J. (1994). Panic disorder: A critical analysis. New York: Guilford Press.

McNally, R. J. (2002). Anxiety sensitivity and panic disorder. Biological Psychiatry, 52, 938-946.

McNally, R. J., \& Foa, E. B. (1987). Cognition and agoraphobia: Bias in the interpretation of threat. Cognitive Therapy and Research, 11, 567-581.

Pilecki, B., Arentoft, A., \& McKay, D. (2011). An evidence-based causal model of panic disorder. Journal of Anxiety Disorders, $25,381-388$

Reiss, S., \& McNally, R. J. (1985). Expectancy model of fear. In S. Reiss \& R. R. Bootzin (Eds.), Theoretical issues in behavior therapy (pp. 107-121). San Diego, CA: Academic Press.

Reiss, S., Peterson, R. A., Gursky, D. M., \& McNally, R. J. (1986). Anxiety sensitivity, anxiety frequency and the prediction of fearfulness. Behaviour Research and Therapy, 24, 1-8.

Richards, J. C., Richardson, V., \& Pier, C. (2002). The relative contributions of negative cognitions and self-efficacy to severity of panic attacks in panic disorder. Behaviour Change, 19, 102-111.

Sandín, B. (2010). Panic Disorder Severity Scale-Self Report (PDSS-SR) (adaptación española). En B. Sandín (Ed.), Trastorno de pánico. Madrid: UNED.
Sandín, B. (2013). DSM-5: ¿Cambio de paradigma en la clasificación de los trastornos mentales? [A paradigm shift in the classification of mental disorders?]. Revista de Psicopatología y Psicología Clínica, 18, 255- 286.

Sandín, B., y Chorot, P. (1989). The incubation theory of fear/ anxiety: Experimental investigation in a human laboratory model of Pavlovian conditioning. Behaviour Research and Therapy, 27, 9-18.

Sandín, B., y Chorot, P. (1991). Trastornos de pánico: Consideraciones sobre su naturaleza, etiología y tratamiento. En G. Buela y V. E. Caballo (Eds.), Manual de psicología clínica aplicada (pp. 305-321). Madrid: Siglo XXI.

Sandín, B., Chorot, P., Santed, M.A., y Valiente, R.M. (2002). Análisis factorial confirmatorio del Índice de Sensibilidad a la Ansiedad para Niños [A confirmatory factor analysis of the Childhood Anxiety Sensitivity Index]. Psicothema, 14, 333-339.

Sandín, B., Chorot, P., y Valiente, R. M. (2012). Transdiagnóstico: Nueva frontera en psicología clínica [Transdiagnostic: A new frontier in clinical psychology]. Revista de Psicopatología y Psicología Clínica, 17, 185-203.

Sandín, B., Chorot, P., Valiente, R.M., y Santed, M.A. (2002). Relación entre la sensibilidad a la ansiedad y el nivel de miedos en niños [Relationship between anxiety sensitivity and fears in children]. Psicología Conductual, 10, 107-120.

Sandín, B., Chorot, P., Valiente, R. M., Sánchez-Arribas, C., \& Santed, M. A. (2004a). Cuestionario de Pánico y Agorafobia (CPA): Características de los ataques de pánico no clínicos [Panic and Agoraphobia Questionnaire: Characteristics of nonclinical panic attacks]. Revista de Psicopatología y Psicología Clínica, 9, 139-161.

Sandín, B., Chorot, P., Valiente, R. M., Santed, M. A., \& Lostao, L. (2004b). Dimensiones de la sensibilidad a la ansiedad: Evidencia confirmatoria de la estructura jerárquica [Dimensions of anxiety sensitivity: Confirmatory evidence of a hierarchic structure]. Revista de Psicopatología y Psicología Clínica, 9, 19-33.

Sandín, B., Rodero, B., Santed, M. A., y García-Campayo, J. (2006). Sucesos vitales estresantes y trastorno de pánico: Relación con el inicio del trastorno, la gravedad clínica y la agorafobia [Stressful life events and panic disorder: Their relation with disorder onset, clinical severity and agoraphobia]. Revista de Psicopatología y Psicología Clínica, 11, 179-190.

Sandín, B., Sánchez-Arribas, C., Chorot, P., \& Valiente, R. M. (2015). Anxiety sensitivity, catastrophic misinterpretations and panic self-efficacy in the prediction of panic disorder severity: Towards a tripartite cognitive model of panic disorder. Behaviour Research and Therapy, 67, 30-40.

Sandín, B., Valiente, R.M., Chorot, P., y Santed, M.A (2005). Propiedades psicométricas del Índice de Sensibilidad a la Ansiedad. Psicothema, 17, 478-483.

Sandín, B., Valiente, R.M., Chorot, P., \& Santed, M.A. (2007). ASI-3: Nueva escala para la evaluación de la sensibilidad a la ansiedad [ASI-3: A new scale for the assessment of anxiety sensitivity]. Revista de Psicopatología y Psicología Clínica, 12, 91-104.

Santacana, M., Fullana, M. A., Bonillo, A., Morales, M., Montoro, M., Rosado, S., ... Bulbena, A. (2014). Psychometric properties of the Spanish self-report version of the Panic Disorder Severity Scale. Comprehensive Psychiatry, 55, 1467-1472. 
Shear, M. K., Brown, T. A., Barlow, D. H., Money, R., Sholomskas, D. E., Woods, S. W., ... \& Papp, L. A. (2006). Multicenter collaborative panic disorder severity scale. American Journal of Psychiatry, 154, 1571-1575.

Taylor, C. B., \& Arnow, B. (1988). The nature and treatment of anxiety disorders. New York: Free Press.

Taylor, S. (1995). Anxiety sensitivity: Theoretical perspectives and recent findings. Behaviour Research and Therapy, 33, 243-258.

Taylor, S. (1999). Anxiety sensitivity: Theory, research, and treatment of the fear of anxiety. Mahwah, NJ: Erlbaum

Taylor, S. (2000). Understanding and treating panic disorder: Cognitive-behavioural approaches. New York: John Wiley \& Sons Ltd.

Taylor, S., \& Cox, B. J. (1998). An expanded anxiety sensitivity index: evidence for a hierarchic structure in a clinical sample. Journal of Anxiety Disorders, 12, 463-483.

Taylor, S., Zvolensky, M. J., Cox, B.J., Deacon, B., Heimberg, R. G., Ledley, D. R., ...Jurado Cardenas, S. (2007). Robust dimensions of anxiety sensitivity: Development and initial validation of the Anxiety Sensitivity Index-3 (ASI-3). Psychological Assessment, 19, 176-188.

Telch, M. J., Brouillard, M., Telch, C. F., Agras, W. S., \& Taylor, C. B. (1989). Role of cognitive appraisal in panic-related avoidance. Behaviour Research and Therapy, 27, 373-383.

Timmerman, M. E., \& Lorenzo-Seva, U. (2011). Dimensionality assessment of ordered polytomous items with parallel analysis. Psychological Methods, 16, 209-220.
Titov, N., Dear, B. F., Johnston, L., \& Terides, M. (2012). Transdiagnostic internet treatment for anxiety and depression. Revista de Psicopatología y Psicología Clínica, 17, 237-260.

Torrents-Rodas, D., Fullana, M. A., Vervliet, B., Treanor, M., Conway, C., Zbozinek, T., \& Craske, M. G. (2015). Maximizar la terapia de exposición: Un enfoque basado en el aprendizaje inhibitorio. Revista de Psicopatología y Psicología Clínica, 1, 1-24.

Valiente, R. M., Sandin, B., \& Chorot, P. (2003). Entrevista para el Diagnóstico de las Trastornos de Ansiedad [Anxiety Disorders Diagnostic Interview] ADIS-M. Madrid: Klinik.

Wenzel, A., Sharp, I. R., Brown, G. K., Greenberg, R. L., \& Beck, A. T. (2006). Dysfunctional beliefs in panic disorder: The panic belief inventory. Behaviour Research and Therapy, 44, 819-833.

Westling, B. E., \& Öst, L. G. (1993). Relationship between panic attack symptoms and cognitions in panic disorder patients. Journal of Anxiety Disorders, 7, 181-194.

Wolpe, J., \& Rowan, V. C. (1988). Panic disorder: A product of classical conditioning. Behaviour Research and Therapy, 26, 441-450.

Zoellner, L. A., Craske, M. G., \& Rapee, R. M. (1996). Stability of catastrophic cognitions in panic disorder. Behaviour Research and Therapy, 34, 399-402.

Zvolensky, M. J., Arrindell, W. A., Taylor, S., Bouvard, M., Cox, B. J., Stewart, S. H., ... \& Eifert, G. H. (2003). Anxiety sensitivity in six countries. Behaviour Research and Therapy, 41, 841-859. 


\section{Anexo I. Cuestionario Abreviado del Trastorno de Pánico (CATP)}

Identificación:

Edad:

Sexo: Hombre $\square$ Mujer $\square$

Fecha:

La crisis de angustia (o ataque de pánico) no es un estado general de ansiedad o nerviosismo que todos experimentamos en ciertos momentos, como por ejemplo asociado a las preocupaciones por el trabajo o los estudios, o la ansiedad normal ante un examen, etc. Una crisis de angustia es una experiencia de intenso miedo o molestia que surge de forma repentina junto a sensaciones especiales como dificultad para respirar, taquicardia, opresión en el pecho, palpitaciones, temblores, sudoración, mareos, y otras reacciones semejantes. Las crisis de angustia suelen ser más frecuente en unas personas que en otras, y también en unas personas se manifiestan de forma más intensa que en otras. Así por ejemplo, mientras que en unos casos la crisis de angustia puede estar acompañada de muchos síntomas, e incluso de intenso miedo o terror, en otros casos las personas sólo experimentan la sensación repentina de molestia y únicamente uno o dos síntomas como los indicados.

1. ¿Cuántas crisis de angustia recuerdas haber tenido en el último año?

$\begin{array}{lllllllllll}0 & 1 & 2 & 3 & 4 & 5 & 6 & 7 & 8 & 9 & 10 \text { o más }\end{array}$

2. ¿Cuántas crisis de angustia has tenido durante el último mes?

$\square$ Ninguna $\square$ Una $\square$ Dos $\square$ Tres $\square$ Más de tres

3. Por favor, indica con qué intensidad sueles experimentar cada uno de los siguientes síntomas durante tus crisis de angustia (rodea el 0 si no has experimentado el síntoma):

1. Palpitaciones, latidos fuertes, o taquicardia

Ninguna
0
0
0
0
0
0
0
0
0
0
0
0
0
0
0

$\begin{array}{cc}\text { Poca } & \text { Bastante } \\ 1 & 2 \\ 1 & 2 \\ 1 & 2 \\ 1 & 2 \\ 1 & 2 \\ 1 & 2 \\ 1 & 2 \\ 1 & 2 \\ 1 & 2 \\ 1 & 2 \\ 1 & 2 \\ 1 & 2 \\ 1 & 2 \\ 1 & 2 \\ 1 & 2\end{array}$

$\begin{array}{cc}\text { Mucha } & \text { Muchísima } \\ 3 & 4 \\ 3 & 4 \\ 3 & 4 \\ 3 & 4 \\ 3 & 4 \\ 3 & 4 \\ 3 & 4 \\ 3 & 4 \\ 3 & 4 \\ 3 & 4 \\ 3 & 4 \\ 3 & 4 \\ 3 & 4 \\ 3 & 4 \\ 3 & 4\end{array}$

4. En general ¿qué gravedad crees que tienen sus crisis de angustia?

$\square$ Ninguna $\square$ Poca $\square$ Bastante $\square$ Mucha $\square$ Muchísima

5. Durante el tiempo que experimentas los primeros síntomas de una posible crisis de angustia, o durante el tiempo que experimentas la crisis de angustia ¿qué sueles pensar o creer que ocurrirá? Por favor, indica la frecuencia con que tienes cada pensamiento ((rodea el número que corresponda):

Pienso que:

1. Puedo tener un ataque al corazón

nunca

pocas veces

varias veces

casi siempre

siempre

2. Podré desmayarme

3. La gente se fijará o se reirá de mí

$\begin{array}{ll}0 & 1 \\ 0 & 1 \\ 0 & 1 \\ 0 & 1 \\ 0 & 1 \\ 0 & 1 \\ 0 & 1 \\ 0 & 1 \\ 0 & 1 \\ 0 & 1 \\ 0 & 1 \\ 0 & 1\end{array}$

4. Mi mente no funcionará bien

5. Puedo perder el control de mis sentidos

6. Será una situación embarazosa

7. No podré pensar o razonar normalmente

8. Puedo tener una trombosis o un derrame cerebral ...........

9. Puedo llamar la atención o hacer el ridículo

10. Me desequilibraré mentalmente

11. Puedo hacer una escena delante de los demás

12. Podré asfixiarme.

$\begin{array}{ll}1 & 2 \\ 1 & 2 \\ 1 & 2 \\ 1 & 2 \\ 1 & 2 \\ 1 & 2 \\ 1 & 2 \\ 1 & 2 \\ 1 & 2 \\ 1 & 2 \\ 1 & 2 \\ 1 & 2 \\ & 2\end{array}$


6. Cuando tienes estos pensamientos que has señalado, ¿hasta qué punto confías en tu propia capacidad para controlarlos y controlar la crisis de angustia, sin necesidad de tomar medicación?

$\square$ Nada $\square$ Poco $\square$ Bastante $\square$ Mucho $\quad \square$ Totalmente

7. Cuando tienes las primeras señales de que va a comenzar una posible crisis de angustia (p.ej., alguna sensación, algún pensamiento, un lugar donde te encuentres, etc.), ¿hasta qué punto confías en tu propia capacidad para evitar tener la crisis, sin necesidad de tomar medicación?
$\square$ Nada
$\square$ Poco
Bastante $\square$ Mucho
Totalmente

8. Cuando ya estás experimentando los síntomas de la crisis, ¿hasta qué punto confías en su propia capacidad para controlar la crisis y lograr que finalice, sin necesidad de tomar medicación?
$\square$ Nada
$\square$ Poco
$\square$ Bastante
$\square$ Mucho
$\square$ Totalmente

9. Cuando notas síntomas de la crisis en alguno de los lugares en que te ocurren habitualmente las crisis, ¿hasta qué punto confías en tu propia capacidad para controlar la crisis sin tener que escapar de esa situación, y sin necesidad de tomar medicación?
$\square$ Nada
$\square$ Poco
Bastante
$\square$ Mucho
$\square$ Totalmente

10. Por favor, indica cuánto temes o te preocupa volver a tener nuevas crisis de angustia o te preocupas por sus posibles $\underline{\text { consecuencias }}$ (p.ej., temor a volverse loco/a, perder el control, etc.):
$\square$ Nada
$\square$ Poco
Bastante
$\square$ Mucho
$\square$ Totalmente

11. ¿Se han producido cambios negativos importantes en tu vida debidos a las crisis, como dejar de hacer ciertas actividades por temor a los síntomas (p.ej., evitar el ejercicio físico, ciertas sustancias o comidas, las relaciones sexuales, etc.) o evitar ciertas situaciones (p.ej., evitar los centros comerciales, los transportes públicos, los cines, estar solo/a fuera de casa)?
$\square$ Nada
$\square$ Poco
$\square$ Bastante $\square$ Mucho
$\square$ Totalmente

12. ¿En general, cuánto están afectando negativamente las crisis de angustia a tus actividades laborales? (p.ej., faltando al trabajo, no atendiendo algunas responsabilidades laborales, rindiendo menos en el trabajo, etc.):
$\square$ Nada
$\square$ Poco
Bastante
$\square$ Mucho
$\square$ Totalmente

13. ¿Cuánto están afectando negativamente las crisis de angustia a tus actividades sociales? (p.ej., no salir con amigos, no asistir a reuniones o fiestas, dejar de ver a algunas personas, perderte relaciones sociales importantes, etc.):
$\square$ Nada
$\square$ Poco
Bastante
Mucho
$\square$ Totalmente

14. ¿Cuánto están afectando negativamente las crisis de angustia a tus actividades y relaciones familiares? (p.ej., no pudiendo atender algunas responsabilidades familiares, creando nuevos problemas en la familia, llevarte peor con la familia, etc.):

$\square$ Nada $\square$ Poco $\square$ Bastante $\square$ Mucho $\square$ Totalmente

(C) 2013 B. Sandín, P. Chorot y R.M. Valiente. Universidad Nacional de Educación a Distancia, Madrid.

Clave de corrección:

EICP Ítem 5. Físico (1, 2, 8, 12), Mental (4, 5, 7 y 10), Social (3, 6, 9 y 11). Total (1-12).

Rango subescalas $=0-16$, rango escala total $=0-48$.

EAP Items 6, 7, 8 y 9. Rango escala $=0-16$.

EGP Items 2, 3 (1-13), 4, 10, 11, 12, 13 y 14. Rango escala $=0-32$.

Items adicionales: Items 1 y 3 ( 14 y 15 ).

Para calcular la puntuación de cada escala (o subescala) se suman las puntuaciones obtenidas en los items de la misma. Las puntuaciones del Ítem 3 (subescala 1-13) se obtienen dividiendo por 13 la suma de los subitems 1-13).

También pueden calcularse las puntuaciones ajustadas al rango de 0-4 (rango de las respuestas) dividiendo la puntuación de cada escala por su número de items. 